Marquette University

e-Publications@Marquette

Physical Therapy Faculty Research and Publications

Physical Therapy, Department of

$4-2019$

\title{
The Effect of a Portable Electrical Muscle Stimulation Device at Home on Muscle Strength and Activation Patterns in Locomotive Syndrome Patients: A Randomized Control Trial
}

\author{
Yuichi Nishikawa \\ Hiroshima University Hospital \\ Kohei Watanabe \\ Chukyo University \\ Shuhei Kawade \\ MTG Co., Ltd. \\ Tetsuya Takahashi \\ Hiroshima University Graduate School of Biomedical and Health Sciences \\ Hiroaki Kimura \\ Hiroshima University Hospital
}

See next page for additional authors

Follow this and additional works at: https://epublications.marquette.edu/phys_therapy_fac

Part of the Physical Therapy Commons

\section{Recommended Citation}

Nishikawa, Yuichi; Watanabe, Kohei; Kawade, Shuhei; Takahashi, Tetsuya; Kimura, Hiroaki; Maruyama, Hirofumi; and Hyngstrom, Allison S., "The Effect of a Portable Electrical Muscle Stimulation Device at Home on Muscle Strength and Activation Patterns in Locomotive Syndrome Patients: A Randomized Control Trial" (2019). Physical Therapy Faculty Research and Publications. 173.

https://epublications.marquette.edu/phys_therapy_fac/173 


\section{Authors}

Yuichi Nishikawa, Kohei Watanabe, Shuhei Kawade, Tetsuya Takahashi, Hiroaki Kimura, Hirofumi Maruyama, and Allison S. Hyngstrom 
Marquette University

e-Publications@Marquette

Physical Therapy Faculty Research and Publications/College of Health Sciences

This paper is NOT THE PUBLISHED VERSION; but the author's final, peer-reviewed manuscript. The published version may be accessed by following the link in th citation below.

Journal of Electromyography and Kinesiology, Vol. 45 (April 2019): 46-52. DOI. This article is (C) Elsevier and permission has been granted for this version to appear in e-Publications@Marquette. Elsevier does not grant permission for this article to be further copied/distributed or hosted elsewhere without the express permission from Elsevier.

\title{
The Effect of a Portable Electrical Muscle Stimulation Device at Home on Muscle Strength and Activation Patterns in Locomotive Syndrome Patients: A Randomized Control Trial
}

\author{
Yuichi Nishikawa \\ Division of Rehabilitation, Department of Clinical Practice and Support, Hiroshima University Hospital, \\ Hiroshima, Japan \\ Department of Physical Therapy, Marquette University, Milwaukee, WI \\ Kohei Watanabe \\ Laboratory of Neuromuscular Biomechanics, School of International Liberal Studies, Chukyo University, \\ Nagoya, Japan \\ Shuhei Kawade \\ MTG Co., Ltd., Nagoya, Japan
}




\title{
Tetsuya Takahashi
}

Department of Clinical Neuroscience and Therapeutics, Hiroshima University Graduate School of Biomedical and Health Sciences, Hiroshima, Japan

\section{Hiroaki Kimura}

Department of Rehabilitation Medicine, Hiroshima University Hospital, Hiroshima, Japan

Hirofumi Maruyama

Department of Clinical Neuroscience and Therapeutics, Hiroshima University Graduate School of Biomedical and Health Sciences, Hiroshima, Japan

\section{Allison Hyngstrom}

\author{
Department of Physical Therapy, Marquette University, Milwaukee, WI
}

\begin{abstract}
The aim of the present study was to quantify the effect of electrical muscle stimulation (EMS) intervention using a portable device on muscle strength and activation patterns in locomotive syndrome. Nineteen women were randomly assigned to the intervention group $(n=10$; age $=71-82$ years) and control group $(n=9$; age $=70-$ 84 years). Participants in the intervention group used a portable EMS device to stimulate the bilateral quadriceps muscles for 8 weeks ( $23 \mathrm{~min} / 5$ days/week). To understand the effects of EMS, the following measurements were made at baseline, 8 weeks, and 12 weeks: locomotive syndrome assessment score, knee extensor strength, vastus lateralis muscle activation patterns during a maximal isometric knee extension contraction using multi-channel surface electromyography, and muscle thickness. The locomotive syndrome assessment, muscle strength, muscle thickness, and muscle activity patterns in the intervention group were significantly different to control after 8 weeks $(p<0.05)$. However, these results were not sustained at 12 weeks. EMS increased locomotor assessment scores, which were accompanied by enhanced muscle strength, increased muscle thickness, and changes in muscle activation patterns in locomotive syndrome patients. These results suggest that EMS is potentially useful for improving muscle neural activation and force output in locomotive syndrome.
\end{abstract}

\section{Keywords}

Locomotive syndrome, Electrical muscle stimulation, Elderly people, Vastus lateralis muscle

\section{Introduction}

The Japanese population is aging rapidly. In 2015, the number of the elderly individuals aged $\geq 65$ years was 33.92 million. This number is increasing and is expected to reach 38.87 million by 2042 (Cabinet, 2016a).

Furthermore, many elderly people require nursing care due to declining health, and the number of individuals who require long-term care is increasing rapidly among the elderly, especially those aged $\geq 75$ years (Cabinet, 2016b). Social benefit expenditure in 2012 was the largest ever, amounting to 1,085,568 million yen (Cabinet, 2016a). Therefore, there is an urgent need for Japan to optimize the health of its aging population to decrease the number of individuals requiring long-term care.

Importantly, health issues related to impaired functional mobility occur in $23.2 \%$ of all patients utilizing longterm care services (falls/fractures, 12.2\%; joint disorders, 11.0\%). Thus, the Japanese Orthopaedic Association proposed the concept of locomotive syndrome in 2007, which they defined as a condition of decreased mobility in activities essential to daily life, such as walking, standing, and climbing stairs, resulting from impairments in 
the musculoskeletal system (Nakamura, 2008, Nakamura, 2011). A test for locomotive syndrome was developed that includes: (1) a stand-up test, (2) a two-step test, and (3) the 25-question risk assessment (Seichi et al., 2012, Yamada et al., 2018). Locomotive syndrome is similar to the concept of frailty except that it is more focused on motor impairments, whereas frailty considers not only physical function but also psychophysiological and social factors (Bandeen-Roche et al., 2015). The motivation for developing the concept of locomotive syndrome was to raise awareness about the importance of preventing the need for nursing care due to dysfunction of the musculoskeletal system among the general public.

Although declines in mobility are often multifactorial, muscle strength is an important factor in the prevention of falls and the progression of orthopedic disorders (e.g., osteoarthritis) (de Zwart et al., 2015, Doherty, 2001). Declines in muscle strength in the elderly are due to both decreased ability to voluntarily activate musculature and muscle atrophy (Ikezoe et al., 2015). Likewise, older individuals who perform muscle strength training improve their physical function. Several studies have shown that training with loads corresponding to $80-85 \%$ of 1 repetition maximum (RM) and beyond (e.g., 1-6 RM) was most effective for increasing maximal dynamic strength (Campos et al., 2002). However, in terms of safety, it is difficult to adapt heavy weight training regimens for elderly people.

Previously, electrical muscle stimulation (EMS) has been effective for increasing muscle mass (Jones et al., 2016) and reversing muscle atrophy caused by bed rest (Gerovasili et al., 2009), immobilization (Dirks et al., 2014), and nerve injury (Marqueste et al., 2004) in older populations. Furthermore, EMS interventions for improving muscle performance and motor unit (MU) activity patterns are widely accepted (Belanger et al., 2000, $\underline{\text { Hirose et al., }}$ 2013, Maffiuletti et al., 2011, Stein et al., 2002, Stevens et al., 2004). Therefore, EMS may be an effective strength training intervention for the elderly and potentially safer than heavy weight training.

Several EMS devices have been developed and used in several settings (e.g., hospital, clinic, and the home). Portable EMS devices are now widely available to the general population (Maffiuletti, 2011). Importantly, EMS interventions can include direct stimulation of the muscle fibers. These portable devices are advantageous because a hospital and/or clinic visit is not required for intervention, making them particularly convenient. However, there are few reports about the effects of portable EMS devices on muscle strength and locomotor activity in older adults.

The aim of this study was to quantify the influence of EMS on neuromuscular performance improvement in patients with locomotive syndrome using a portable device.

The spatial and temporal muscle activation pattern within muscles has been recently assessed through the spatial distribution patterns of muscle activation using multi-channel surface electromyography(SEMG) (Farina et al., 2008, Merletti et al., 2008). Furthermore, multi-channel SEMG can measure some muscle features (e.g., the heterogeneity of the activation changes) that are difficult to detect by other methods. Therefore, we performed the assessment of muscle activation patterns using a multi-channel SEMG method. We hypothesized that EMS interventions would enhance muscle strength, increase locomotion performance, and influence muscle activity patterns in locomotive syndrome patients.

\section{Materials and methods}

\subsection{Participants}

A total of 19 women were randomly assigned to the intervention group $(n=10$; age $=71-82$ years; height $=151.9 \pm 4.3 \mathrm{~cm}$; body mass $=52.9 \pm 5.0 \mathrm{~kg})$ and control group $(n=9$; age $=70-84$ years; height $=149.1 \pm 2.3 \mathrm{~cm}$; body mass $=48.7 \pm 5.2 \mathrm{~kg}$ ). Our previous study showed that compared with healthy young males, healthy females exhibited greater differences in the spatial distribution patterns of muscle 
activation using multi-channel SEMG during sustained isometric contractions (Nishikawa et al., 2017). This finding suggests that it is necessary to differentiate between males and females when assessing muscle activation pattern using multi-channel SEMG. Therefore, we included only women in the current study. The inclusion criteria were the ability to independently perform activities of daily living and a diagnosis of locomotive syndrome. Locomotive syndrome was assessed by three tests (Supplementary Fig. 1and Supplementary Table 1): (1) stand-up test (cannot stand up from sitting on a 40-cm stool with one leg); (2) two-step test $(<1.3)$; and (3) the 25-question risk assessment ( $>7$ ) (Seichi et al., 2012, Yamada et al., 2018). The exclusion criteria were neuromuscular disease, cardiovascular disease, and diabetes mellitus. All procedures were performed in accordance with the Declaration of Helsinki and were approved by Hiroshima University's Committee on Ethics in Research (approval no. C-151). All participants signed an informed consent form and consented to the publication of this work. The following measurements were made at baseline, 8 weeks, and 12 weeks for all participants: muscle strength, ultrasound measurements, and multi-channel SEMG measurements (Fig. 1).

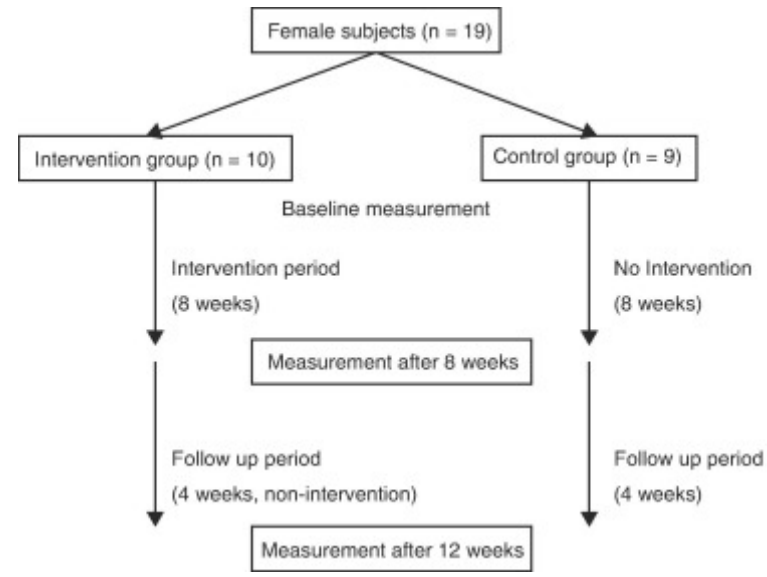

Fig 1. Time course of the study.

\subsection{Experimental design}

In this study, we performed single-blinded intervention (participants). Participants in the intervention group underwent EMS of the quadriceps muscle of both legs for 8 weeks; then, they were followed-up for 4 weeks. A portable EMS device (Sixpad Body fit; MTG Ltd, Nagoya, Japan) was used 5 days per week. The device was positioned at the mid-point of the femur. Muscles were stimulated at an intensity of $4.85 \mathrm{~mA}$ (frequency $=20 \mathrm{~Hz}$, pulse shape = square wave, pulse duration $=100 \mu \mathrm{s}$, pulse period $=50 \mathrm{~ms}$, size $=122 \mathrm{~mm} \times 244 \mathrm{~mm}$, material of electrode = conductive silver and carbon ink, interelectrode distance $=500 \mathrm{~mm}$, Supplementary Fig. 2) for 23 min once per day. Participants in the intervention group underwent EMS intervention in a sitting position at home. Participants were instructed not to actively contract muscles during the stimulation protocol. Those in the control group did not undergo any intervention. Although the EMS intervention was applied to both legs, the following test measurements were made on a single test leg (e.g., dominant side). To understand the effects of EMS, the following measurements were made at baseline, 8 weeks, and 12 weeks: maximal voluntary knee extensor contraction strength, vastuslateralis muscle activation patterns during a submaximal isometric knee extension using multi-channel SEMG, muscle thickness, and locomotive syndrome scores.

\subsection{Maximal voluntary strength}

All participants performed maximal voluntary contractions (MVCs) during isometric knee extension at baseline, 8 weeks, and 12 weeks. Isometric knee extension was performed using a Biodex system (Biodex System 3; Biodex Medical Systems, Shirley, NY, USA) (Watanabe et al., 2012b). During contraction, both the hip and knee extension angles were fixed at $90^{\circ}$, respectively. The MVC involved a gradual increase in knee extension torque 
exerted by the knee extensor muscles from 0 to maximum over $3 \mathrm{~s}$, with the maximum torque held for $2 \mathrm{~s}$. The participants performed at least two MVC trials with $>120 \mathrm{~s}$ of rest between trials, and a warm up for $10 \mathrm{~min}$, including indoor walking and lower limb stretching before MVC measurement. The highest MVC torque was used to calculate the MVC torque and target torque for sustained contractions. This assessment was performed at baseline, 8 weeks, and 12 weeks.

\subsection{Ultrasound measures of muscle thickness}

The thickness of the dominant vastus lateralis $(\mathrm{VL})$ muscle at the center of the line between the superior lateral edge of the patella and greater trochanter protuberance was obtained from ultrasound images using a linear array probe with a frequency of $7.5 \mathrm{MHz}$ (Noblus; Hitachi Aloka Medical, Tokyo, Japan) (Nishikawa et al., 2017, Watanabe et al., 2012b). We calculated the percent change from baseline in muscle thickness at 8 weeks and 12 weeks.

\subsection{SEMG recording}

The participant performed a maximum isometric knee extension contraction. During the maximal contraction, multi-channel SEMG signals were detected from the dominant VL muscle using a semi-disposable grid of 64 electrodes (ELSCH064RS3; OT Bioelettronica, Torino, Italy) according to the same procedure used in previous studies (Nishikawa et al., 2017, Watanabe et al., 2012b). The grid consisted of 13 columns and five rows of electrodes (diameter, $1 \mathrm{~mm}$; inter-electrode distance, $8 \mathrm{~mm}$ in each direction), with one missing electrode in the upper left corner. The participants hair was removed, the skin was cleaned with alcohol, and the electrode was attached to the skin with a bi-adhesive sheet (KITAD064; OT Bioelettronica) after applying conductive paste (Elefix Z-181BE; NIHON KOHDEN, Tokyo, Japan) corresponding to the placement of the electrodes. The center of the electrode grid was attached at the center of the line between the superior lateral edge of the patella and the greater trochanter protuberance. The columns of the electrode grid were placed parallel to the longitudinal axis of the VL muscle. The site of the missing electrode was placed proximal to the VL muscle. A reference electrode was attached at the anterior superior iliac spine (see Fig. 2). All procedures were performed by the same investigator.
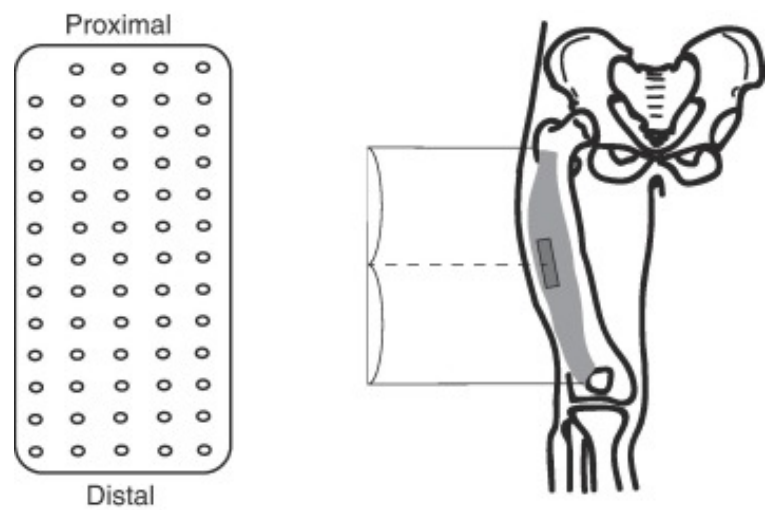

Fig 2. The grid consisted of 13 rows and five columns of electrodes (diameter, $1 \mathrm{~mm}$; inter-electrode distance, $8 \mathrm{~mm}$ in each direction), with one missing electrode in the upper left corner. The multi-channel surface electromyography electrode grid was placed on the vastus lateralis muscleat the center of the line between the superior lateral edge of the patella and the greater trochanter protuberance. A reference electrode was attached at the anterior superior iliac spine.

Monopolar multi-channel SEMG signals were amplified by a factor of 1000 , sampled at $2048 \mathrm{~Hz}$ per channel, and converted to digital data using a 12-bit analog-to-digital converter (EMG-USB2+; OT Bioelettronica). The recorded monopolar multi-channel SEMG signals were off-line bandpass-filtered $(10-500 \mathrm{~Hz})$ and transferred to software for analysis (MATLAB 2018a; Math Works GK, Natick, MA, USA). Bipolar multi-channel SEMG signals $(n=59)$ along the columns were divided from the 64 electrodes. To calculate the root mean square (RMS) of 
multi-channel SEMG signals, the signals were sampled over $1 \mathrm{~s}$ during the sustained phase after peak torque was achieved. To control for inter-participant variability, we normalized the RMS measures to the values obtained at baseline (0 weeks).

To characterize the heterogeneity in the spatial multi-channel SEMG potential distribution, we determined the modified entropy and the coefficient of variation ( $\mathrm{CoV}$ ) of spatial RMS estimates. The modified entropy of the spatial distribution of the SEMG amplitude was calculated for 59 RMS values (in space) of single differential signals computed over a 1-s period taken at the time of the contraction during the isometric sustained contraction. Using the methods of Farina et al. (2008), modified entropy was calculated for 59 absolute RMS values at MVC. The CoV of spatial RMS estimates was defined as the quotient of the standard deviation of the 59 RMS measurements and the average of 59 RMS measurement. A decrease in the modified entropy and an increase in the CoV of spatial RMS estimates indicated increased heterogeneity in the spatial multi-channel SEMG potential distribution within the electrode grid (Watanabe et al., 2012a). Recent studies have quantified the spatial distribution pattern of SEMG to estimate the spatial and temporal activation pattern within muscles using multi-channel SEMG (Holtermann et al., 2008, Watanabe et al., 2015). We calculated the percent change in $\mathrm{CoV}$ and entropy measures from baseline at 8 weeks and 12 weeks.

\subsection{Statistical analysis}

Statistical analyses were performed using JMP statistical software version 12 (SAS Institute, Inc., Cary, NC, USA). The continuous data are presented as the mean \pm standard deviation or the median (minimum, maximum). Before the analysis, the normal distribution of data was confirmed using the Shapiro-Wilk test. Age, height, body mass, muscle thickness, two-step test, 25-question risk assessment, and MVC torque at baseline were compared between the intervention and control groups using unpaired $t$-tests. The $\Delta$ maximal knee extension torque, $\Delta$ muscle thickness, $\Delta$ modified entropy, and $\Delta \mathrm{CoV}$ of RMS were analyzed using the Wilcoxon signed-rank test between 8 weeks and 12 weeks for each group. The Mann-Whitney $U$ test was used at each time point (e.g., 8 weeks and 12 weeks) to compare the values described above between the intervention and control groups. Bonferroni-adjusted $p$ values were used $(p<0.01)$.

\section{Results}

The general characteristics of the participants are presented in Table 1. There were no significant differences between groups in terms of the anthropometric parameters.

Table 1. Characteristics of subjects.

\begin{tabular}{|l|l|l|}
\hline Variables & Intervention group $(\mathrm{n}=10)$ & Control group $(\mathrm{n}=9)$ \\
\hline Age, years & $75.6 \pm 3.7$ & $77.3 \pm 3.9$ \\
\hline Height, $\mathrm{cm}$ & $151.9 \pm 4.3$ & $149.1 \pm 2.3$ \\
\hline Body mass, kg & $52.9 \pm 5.0$ & $48.7 \pm 5.2$ \\
\hline Knee extension torque, Nm & $70.3 \pm 16.5$ & $69.4 \pm 10.1$ \\
\hline
\end{tabular}

Data are presented as the mean \pm SD.

The stand-up test did not show improvement between each period (e.g., baseline, 8 weeks, and 12 weeks) in each group. The two-step test and 25-question risk assessment did not show significant differences between groups at baseline ( $p=0.732$ and $p=0.247$, respectively). In particular, question 15 (regarding gait distance) and question 18 (regarding going out using public transportation) showed improvement compared with baseline in the intervention group (Fig. 4). The percent change in two-step test and 25-question risk assessment scores were significantly greater in the intervention group $(p=0.0275$ and $p=0.0156)$. Furthermore, the $\Delta$ two-step test and 25-question risk assessment in the intervention group showed significant improvement compared with 
the control group at 8 weeks (median (min-max), 6.14 (0.46-19.9) \% vs. $0.14(-1.35$ to 0.19$) \%$ and -28.6 ( -50 to $12.5) \%$ vs. 0 ( -12.5 to 16.7$) \%, p=0.0003$, respectively, Fig. 3 ).

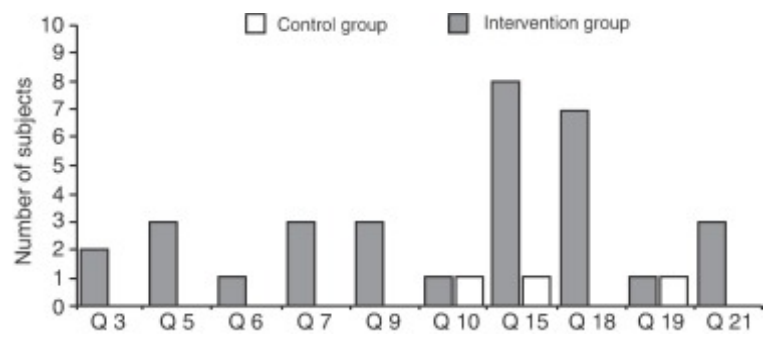

Fig 3. Comparison of $\Delta$ two-step test (A) and $\Delta 25$-question risk assessment between 8 weeks and 12 weeks (D). ${ }^{*} p<0.01$, significant difference.
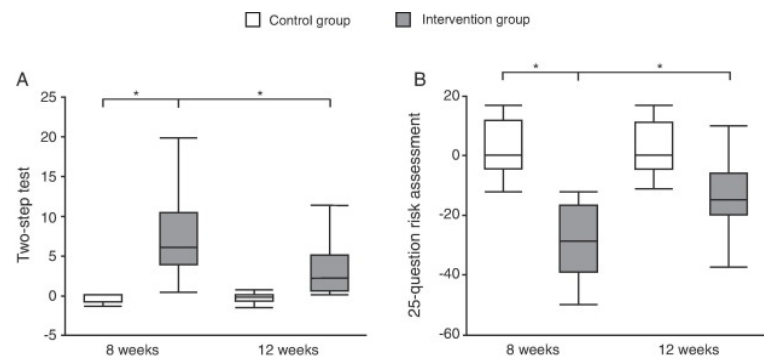

Fig 4. Number of participants with improvement in 25-question risk assessment from baseline to 8 weeks in each group.

The percent change in knee extension torque was significantly greater in the intervention group $(p=0.0003, \underline{\text { Fig }}$. $\underline{5}$ B). The $\Delta$ knee extension torque showed a significant decrease between 8 weeks and 12 weeks $(p=0.0020)$ for the intervention group. On the other hand, the control group did not show a significant difference between 8 and 12 weeks (Fig. $5 \mathrm{~A}$ ). The $\Delta$ muscle thickness value showed a decrease between 8 weeks and 12 weeks $(p=0.0020)$ for the intervention group ( $\underline{\text { Fig. } 5}$ B). However, the control group showed no significant difference at each time point. Furthermore, $\Delta$ muscle thickness at 8 weeks in the intervention group was significantly higher than that in the control group ( $p=0.0006$, Fig. 5 B).

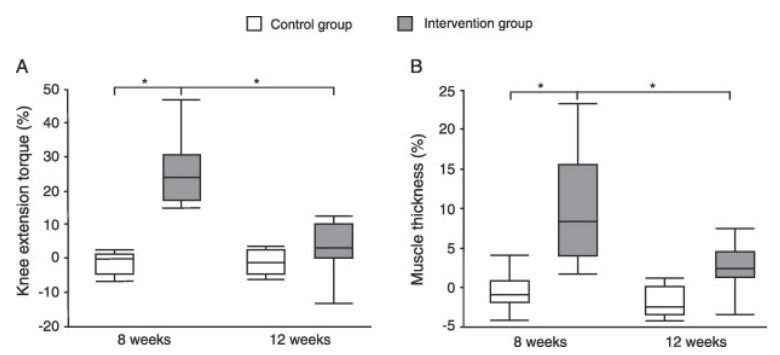

Fig 5. Comparison of $\Delta$ knee extension torque (A) and $\Delta$ muscle thickness between 8 weeks and 12 weeks (B) for each group. ${ }^{*} p<0.01$, significant difference.

The $\Delta$ modified entropy significantly increased between 8 weeks and 12 weeks $(p=0.0002)$ in the intervention group (Fig. 6 A) but not in the control group $(p=0.3314)$. Furthermore, the intervention group showed a significant decrease compared with the control group at 8 weeks $(p=0.0003)$. The $\Delta$ CoV of RMS exhibited a significant difference between 8 weeks and 12 weeks in the intervention group ( $p=0.0002$, Fig. 6 B) but not in the control group $(p=0.6588)$. Furthermore, the intervention group showed a significant increase compared with the control group at 8 weeks $(p=0.0017)$. 

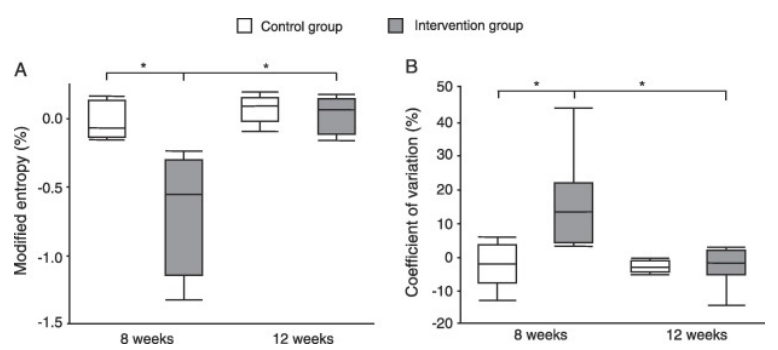

Fig. 6. Comparison of $\Delta$ modified entropy (A) and $\Delta$ coefficient of variation (B) between 8 weeks and 12 weeks. ${ }^{*} p<0.01$, significant difference.

\section{Discussion}

The present study examined the effects of EMS on locomotive syndrome assessment scores, muscle strength, muscle activation patterns, and muscle thickness in locomotive syndrome patients. The primary results of the present study were that EMS of the quadriceps improved locomotive syndrome assessment scores, increased muscle strength, and led to more inhomogeneity in spatial muscle distribution patterns. In addition, muscle strength and muscle activation patterns could not be sustained without EMS intervention.

The results of the present study showed that locomotive syndrome assessment scores (e.g., two-step test and 25-question risk assessment), muscle strength, and muscle thickness were significantly enhanced by EMS in the intervention group ( $\mathrm{Fig} \mathrm{3}$, Fig 4, Fig 5). Furthermore, many participants in the intervention group showed improvements in gait distance and going out using public transportation in the 25-question risk assessment. These results suggested that EMS intervention is effective not only for muscle performance but also for activities of daily living. In contrast, the stand-up test did not show improvement between each period (e.g., baseline, 8 weeks, and 12 weeks) in each group. It is widely known that EMS intervention can improve muscle performance (Belanger et al., 2000, Hirose et al., 2013, Maffiuletti et al., 2011, Stein et al., 2002, Stevens et al., 2004). Furthermore, many previous studies reported that improvements in muscle strength and thickness occurred after EMS interventions (Boutelle et al., 1985, Broekmans et al., 2011, 1988). These previous findings are in accordance with the results of the present study, which compared a control group with an intervention group to show significantly enhanced strength and muscle thickness in this special population. These findings suggest that EMS is an effective treatment for individuals with locomotive syndrome. However, participants in the intervention group only received stimulation to their quadriceps muscle. Because locomotor tasks require coordination and strength in several muscles (e.g., gastrocnemius $\underline{\text { muscle, }}$ tibialis anterior muscle, and hamstrings) for tasks such as standing (Donath et al., 2016, Kurz et al., 2018), future studies should examine the effects of EMS on other leg muscles.

In addition to increases in maximal strength-generating capability, the results of the present study showed that spatial muscle distribution patterns were changed by EMS interventions. We used modified entropy and CoV of spatial RMS estimates to assess muscle activation patterns. A decrease in the modified entropy and an increase in the CoV of spatial RMS estimates is consistent with increased heterogeneity in the spatial multi-channel SEMG potential distribution within the electrode grid (Watanabe, 2012a). A previous study reported that a greater decrease in entropy positively correlated with endurance time for low-intensity, isometric contraction ( $\underline{\text { Farina, }}$ 2008). In general, females are less fatigable than males during low-intensity, sustained isometric contraction (Hunter and Enoka, 2001). Our previous study reported that compared with healthy young males, healthy females exhibited more changes of modified entropy and CoV of spatial RMS estimates (Nishikawa, 2017). These findings suggest that redistribution of muscle activation plays a key role in prolonging muscular fatigue during sustained contraction (Farina, 2008). Therefore, the results of the present study suggested that EMS interventions might have a positive influence on muscle activity in $\mathrm{VL}$ muscles. 
The present study showed decreased force and neuromuscular function(e.g., locomotive syndrome assessment scores, muscle strength, and EMG variables) at the end of the follow up period (12 w) in the intervention group (Fig 5, Fig. 6). Therefore, our results might only influence early neural adaptations enhancing muscle activation by EMS intervention. Previous studies have reported a decrease in muscle strength and RMS values during the detraining period caused by inactivity and/or without EMS intervention ( 1989 , Natsume et al., 2015). Natsume et al. reported that $2 \mathrm{w}$ of EMS detraining reduced muscle strength $(-6.8 \%)$ and muscle thickness $(-3.0 \%)$ in healthy young adults (Natsume, 2015). These previous findings are in accordance with the results of this study showing that physical and neuromuscular function return to baseline values during follow up in the intervention group. These findings suggested that it is necessary to continue the EMS intervention to prevent a decrease in physical and neuromuscular function.

In the present study, we demonstrated the feasibility of using a small portable EMS device. Many previous studies have performed EMS interventions at a hospital and/or laboratory. Therefore, participants had to leave their homes for each experimental course. However, during our study, they did not have to leave their homes because they used a portable device.

The present study has several limitations. First, participants performed the intervention independently at home. Therefore, there might have been some variability in the application of the EMS and compliance. To help control for these factors, we checked compliance with the intervention procedures by telephoning participants every month (Supplementary Fig. 3). Second, we did not compare portable and non-portable devices to determine which might have greater effects on muscle strength and activation patterns. Similar to previous studies using non-portable EMG interventions, we did see that muscle strength and activity patterns improved and/or changed with the use of the portable device and returned to baseline values during the follow-up periods. Future studies will compare the effectiveness of portable versus non-portable EMG devices.

The present study showed that improvement of muscle performance and instrumental activities of daily living in locomotive syndrome patients were induced by EMS intervention. Therefore, we would like to extend healthy life expectancies using this method. Future studies using portable and non-portable EMS devices and intervention combinations (e.g., EMS and exercise training vs. only EMS vs. only exercise training) and doubleblinded study designs (tester and participant) are needed to gain a better understanding of the most effective methods to treat locomotive syndrome patients.

\section{Conclusions}

We investigated the effects of EMS on muscle strength and activation patterns using a portable EMS device. The present study showed increased muscle strength, more inhomogeneity of spatial muscle distribution patterns, and that muscle strength and muscle activation patterns cannot be sustained without EMS intervention. These results suggest that a portable EMS device enhances muscle strength and changes muscle activation patterns in individuals with locomotive syndrome.

\section{Conflicts of interest}

The authors declare no conflict of interest and that no companies or manufacturers will benefit from the results of this study.

\section{Acknowledgments}

This research was supported in part by research grants from Hiroshima University Hospital, Japan (grant numbers $\underline{30 K 172}$ ) and the Japanese Society for Musculoskeletal Medicine (grant number 3010526). 


\section{Conflict of interest}

The authors declare no conflict of interest and that no companies or manufacturers will benefit from the results of this study.

\section{Appendix A. Supplementary material}

A

Supplementary Fig.1 Nishikawa Y

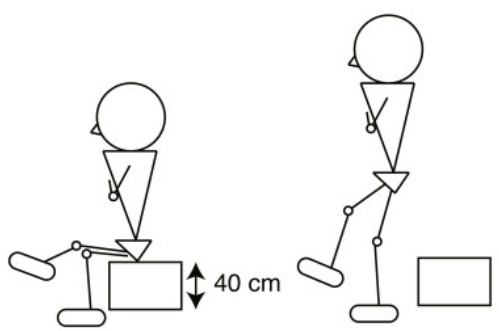

B

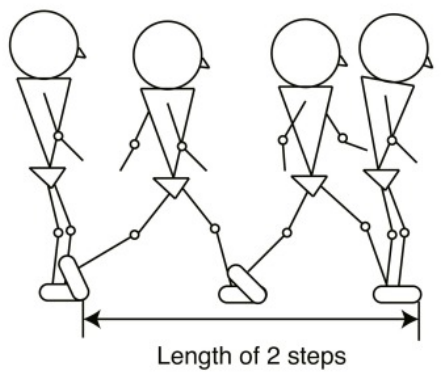

Length of 2 steps $(\mathrm{cm}) \div$ height $(\mathrm{cm})=2$ step value

The following are the Supplementary data to this article:

Supplementary Fig. 1. (A) Stand-up test. Participants were asked to stand from a 40-cm stool with one leg. If they succeeded in standing and maintaining the posture for $3 \mathrm{~s}$, the trial was considered complete. (B) Two-step test. This test measured the maximum stride length of two steps from the standing posture. The two-step test score is the length of the two steps $(\mathrm{cm}) \div$ the participant's height $(\mathrm{cm})$.

Supplementary Fig.2 Nishikawa $Y$

Unit (front)

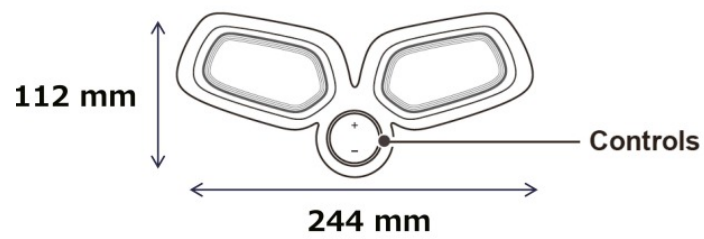

Unit (rear)

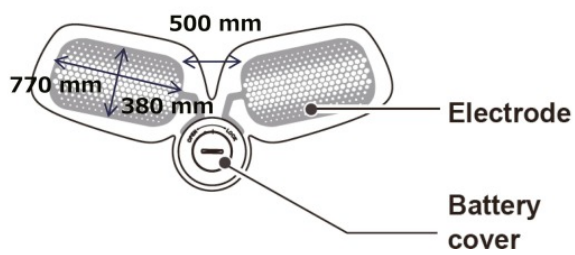

Supplementary Fig. 2. Size and design of a portable electrical muscle stimulation device. 


\begin{tabular}{|c|c|c|c|c|c|c|c|}
\hline & SUN & MON & TUE & WED & тни & FRI & SAT \\
\hline $1 w$ & & 0 & 0 & 0 & 0 & 0 & \\
\hline $2 w$ & & & & & & & \\
\hline $3 w$ & & & & & & & \\
\hline $4 w$ & & & & & & & \\
\hline $5 w$ & & & & & & & \\
\hline 6w & & & & & & & \\
\hline $7 \mathrm{w}$ & & & & & & & \\
\hline $8 w$ & & & & & & & \\
\hline
\end{tabular}

Supplementary Fig. 3. Intervention check sheet. When participants in the intervention group performed electrical muscle stimulation, they drew a circle or check on the sheet.

Research data for this article

Data not available / Data will be made available on request

\section{References}

Bandeen-Roche et al., 2015 K. Bandeen-Roche, C.L. Seplaki, J. Huang, B. Buta, R.R. Kalyani, R. Varadhan, et al. Frailty in older adults: a nationally representative profile in the United States J. Gerontol. A Biol. Sci. Med. Sci., 70 (11) (2015), pp. 1427-1434

Belanger et al., 2000 M. Belanger, R.B. Stein, G.D. Wheeler, T. Gordon, B. Leduc Electrical stimulation: can it increase muscle strength and reverse osteopenia in spinal cord injured individuals? Arch. Phys. Med. Rehabil., 81 (8) (2000), pp. 1090-1098

Boutelle et al., 1985 D. Boutelle, B. Smith, T.R. Malone A strength study utilizing the electro-stim 180 J. Orthop. Sports Phys. Ther., 7 (2) (1985), pp. 50-53

Broekmans et al., 2011 T. Broekmans, M. Roelants, P. Feys, G. Alders, D. Gijbels, I. Hanssen, et al. Effects of longterm resistance training and simultaneous electro-stimulation on muscle strength and functional mobility in multiple sclerosis Mult Scler., 17 (4) (2011), pp. 468-477

Cabinet, 2016a Cabinet Office, 2016a. Chapter 1. Situation on Aging. Goverment of Japan.

Cabinet, 2016b Cabinet Office, 2016b. Chart 1-2-13. Healthy Life Expectancy at Birth and Life Expectancy at Birth Goverment of Japan.

Campos et al., 2002 G.E. Campos, T.J. Luecke, H.K. Wendeln, K. Toma, F.C.Hagerman, T.F. Murray, et al. Muscular adaptations in response to three different resistance-training regimens: specificity of repetition maximum training zones Eur. J. Appl. Physiol., 88 (1-2) (2002), pp. 50-60

de Zwart et al., 2015 A.H. de Zwart, M. van der Esch, M.A. Pijnappels, M.J.Hoozemans, M. van der Leeden, L.D. Roorda, et al. Falls associated with muscle strength in patients with knee osteoarthritis and self-reported knee instability J. Rheumatol., 42 (7) (2015), pp. 1218-1223

Dirks et al., 2014 M.L. Dirks, B.T. Wall, T. Snijders, C.L. Ottenbros, L.B. Verdijk, L.J. van Loon Neuromuscular electrical stimulation prevents muscle disuse atrophy during leg immobilization in humans Acta Physiol. (Oxf)., 210 (3) (2014), pp. 628-641

Doherty, 2001 M. Doherty Risk factors for progression of knee osteoarthritis Lancet, 358 (9284) (2001), pp. $775-776$

Donath et al., 2016 L. Donath, E. Kurz, R. Roth, L. Zahner, O. Faude Leg and trunk muscle coordination and postural sway during increasingly difficult standing balance tasks in young and older adults Maturitas, 91 (2016), pp. 60-68 
Farina et al., 2008 D. Farina, F. Leclerc, L. Arendt-Nielsen, O. Buttelli, P.Madeleine The change in spatial distribution of upper trapezius muscle activity is correlated to contraction duration J. Electromyogr. Kinesiol., 18 (1) (2008), pp. 16-25

Gerovasili et al., 2009 V. Gerovasili, K. Stefanidis, K. Vitzilaios, E. Karatzanos, P.Politis, A. Koroneos, et al. Electrical muscle stimulation preserves the muscle mass of critically ill patients: a randomized study Crit. Care, 13 (5) (2009), p. R161

Hakkinen and Komi, 1983 K. Hakkinen, P.V. Komi Electromyographic changes during strength training and detraining Med. Sci. Sports Exerc., 15 (6) (1983), pp. 455-460

Hasegawa et al., 2011 S. Hasegawa, M. Kobayashi, R. Arai, A. Tamaki, T.Nakamura, T. Moritani Effect of early implementation of electrical muscle stimulation to prevent muscle atrophy and weakness in patients after anterior cruciate ligament reconstruction J. Electromyogr. Kinesiol., 21 (4) (2011), pp. 622-630

Hirose et al., 2013 T. Hirose, T. Shiozaki, K. Shimizu, T. Mouri, K. Noguchi, M.Ohnishi, et al. The effect of electrical muscle stimulation on the prevention of disuse muscle atrophy in patients with consciousness disturbance in the intensive care unit J. Crit. Care, 28 (4) (2013) 536.e1-7

Holtermann et al., 2008 A. Holtermann, C. Grönlund, J. Stefan Karlsson, K. Roeleveld Spatial distribution of active muscle fibre characteristics in the upper trapezius muscle and its dependency on contraction level and duration J. Electromyogr. Kinesiol., 18 (3) (2008), pp. 372-381

Hunter and Enoka, 2001 S.K. Hunter, R.M. Enoka Sex differences in the fatigability of arm muscles depends on absolute force during isometric contractions J. Appl. Physiol., 91 (6) (2001), pp. 2686-2694

Ikezoe et al., 2015 T. Ikezoe, M. Nakamura, H. Shima, Y. Asakawa, N. Ichihashi Association between walking ability and trunk and lower-limb muscle atrophy in institutionalized elderly women: a longitudinal pilot study J. Physiol. Anthropol., 34 (2015), p. 31

Jones et al., 2016 S. Jones, W.D. Man, W. Gao, I.J. Higginson, A. Wilcock, M.Maddocks Neuromuscular electrical stimulation for muscle weakness in adults with advanced disease Cochrane Database Syst. Rev., 10 (2016), p. Cd009419

Kurz et al., 2018 E. Kurz, O. Faude, R. Roth, L. Zahner, L. Donath Ankle muscle activity modulation during singleleg stance differs between children, young adults and seniors Eur. J. Appl. Physiol., 118 (2) (2018), pp. 239-247

Lai et al., 1988 H.S. Lai, G.D. Domenico, G.R. Strauss The effect of different electro-motor stimulation training intensities on strength improvement Aust. J. Physiother., 34 (3) (1988), pp. 151-164

Maffiuletti et al., 2011 N.A. Maffiuletti, M.A. Minetto, D. Farina, R. Bottinelli Electrical stimulation for neuromuscular testing and training: state-of-the art and unresolved issues Eur. J. Appl. Physiol., 111 (10) (2011), pp. 2391-2397

Marqueste et al., 2004 T. Marqueste, J.R. Alliez, O. Alluin, Y. Jammes, P.Decherchi Neuromuscular rehabilitation by treadmill running or electrical stimulation after peripheral nerve injury and repair J. Appl. Physiol. (1985), 96 (5) (2004), pp. 1988-1995

Merletti et al., 2008 R. Merletti, A. Holobar, D. Farina Analysis of motor units with high-density surface electromyography J. Electromyogr. Kinesiol., 18 (6) (2008), pp. 879-890

Nakamura, 2008 K. Nakamura A "super-aged" society and the "locomotive syndrome" J. Orthop. Sci., 13 (1) (2008), pp. 1-2

Nakamura, $2011 \mathrm{~K}$. Nakamura The concept and treatment of locomotive syndrome: its acceptance and spread in Japan J. Orthop. Sci., 16 (5) (2011), pp. 489-491

Narici et al., 1989 M.V. Narici, G.S. Roi, L. Landoni, A.E. Minetti, P. Cerretelli Changes in force, cross-sectional area and neural activation during strength training and detraining of the human quadriceps Eur. $J$. Appl. Physiol. Occup. Physiol., 59 (4) (1989), pp. 310-319

Natsume et al., 2015 T. Natsume, H. Ozaki, A.I. Saito, T. Abe, H. Naito Effects of electrostimulation with blood flow restriction on muscle size and strength Med. Sci. Sports Exerc., 47 (12) (2015), pp. 2621-2627 
Nishikawa et al., 2017 Y. Nishikawa, K. Watanabe, T. Takahashi, N. Hosomi, N.Orita, Y. Mikami, et al. Sex differences in variances of multi-channel surface electromyography distribution of the vastus lateralis muscle during isometric knee extension in young adults Eur. J. Appl. Physiol., 117 (3) (2017), pp. 583589

Seichi et al., 2012 A. Seichi, Y. Hoshino, T. Doi, M. Akai, Y. Tobimatsu, T. Iwaya Development of a screening tool for risk of locomotive syndrome in the elderly: the 25-question Geriatric Locomotive Function Scale $J$. Orthop. Sci., 17 (2) (2012), pp. 163-172

Stein et al., 2002 R.B. Stein, S.L. Chong, K.B. James, A. Kido, G.J. Bell, L.A.Tubman, et al. Electrical stimulation for therapy and mobility after spinal cord injury Prog. Brain Res., 137 (2002), pp. 27-34

Stevens et al., 2004 J.E. Stevens, R.L. Mizner, L. Snyder-Mackler Neuromuscular electrical stimulation for quadriceps muscle strengthening after bilateral total knee arthroplasty: a case series J. Orthop. Sports Phys. Ther., 34 (1) (2004), pp. 21-29

Watanabe et al., 2012a K. Watanabe, M. Kouzaki, R. Merletti, M. Fujibayashi, T.Moritani Spatial EMG potential distribution pattern of vastus lateralis muscle during isometric knee extension in young and elderly men J. Electromyogr. Kinesiol., 22 (1) (2012), pp. 74-79

Watanabe et al., $2015 \mathrm{~K}$. Watanabe, M. Kouzaki, T. Moritani Spatial EMG potential distribution of biceps brachii muscle during resistance training and detraining Eur. J. Appl. Physiol., 115 (12) (2015), pp. 2661-2670

Watanabe et al., 2012b K. Watanabe, T. Miyamoto, Y. Tanaka, K. Fukuda, T.Moritani Type 2 diabetes mellitus patients manifest characteristic spatial EMG potential distribution pattern during sustained isometric contraction Diabetes Res. Clin. Pract., 97 (3) (2012), pp. 468-473

Yamada et al., 2018 K. Yamada, S. Muranaga, T. Shinozaki, K. Nakamura, S.Tanaka, T. Ogata Age independency of mobility decrease assessed using the Locomotive Syndrome Risk Test in elderly with disability: a cross-sectional study BMC Geriatr., 18 (1) (2018), p. 28 